\title{
Comparison of the radiomics-based predictive models using machine learning and nomogram for epidermal growth factor receptor mutation status and subtypes in lung adenocarcinoma
}

\section{Yusuke Kawazoe}

Yamaguchi University: Yamaguchi Daigaku

Takehiro Shiinoki ( $\sim$ shiinoki@yamaguchi-u.ac.jp)

Yamaguchi University: Yamaguchi Daigaku https://orcid.org/0000-0002-0246-8489

Koya Fujimoto

Yamaguchi University: Yamaguchi Daigaku

\section{Yuki Yuasa}

Yamaguchi University: Yamaguchi Daigaku

\section{Tsunahiko Hirano}

Yamaguchi University: Yamaguchi Daigaku

\section{Kazuto Matsunaga}

Yamaguchi University: Yamaguchi Daigaku

\section{Hidekazu Tanaka}

Yamaguchi University: Yamaguchi Daigaku

\section{Research Article}

Keywords: computed tomography, EGFR mutation, machine learning, nomogram, radiomics

Posted Date: February 7th, 2022

DOl: https://doi.org/10.21203/rs.3.rs-1176316/v1

License: (9) (i) This work is licensed under a Creative Commons Attribution 4.0 International License. Read Full License 


\section{Abstract}

Introduction: The purpose of this study is to develop the predictive models for epidermal growth factor receptor (EGFR) mutation status and subtypes [exon 21-point mutation (L858R) and exon 19 deletion mutation (19Del)] and validate the clinical effectiveness.

Methods: Total 172 patients with lung adenocarcinoma were enrolled. The analysis of variance and the least absolute shrinkage were used feature selection from plain computed tomography images. Then, radiomic score (rad-score) was calculated for the training and test cohorts. Two machine learning (ML) models with 5-fold were applied to construct the predictive models with rad-score and clinical features and evaluated by the area under the curve (AUC) of receiver operating characteristic curve. The nomogram was developed using rad-score and clinical features, then evaluated by the C-index. Finally, decision curve analysis (DCA) was performed using the best ML and nomogram models.

Results: The AUC of the best ML models and the C-index of the nomogram models were $0.82,0.73$ and $0.84,0.78$ in the EGFR mutation groups, $0.83,0.83$ and $0.83,0.80$ in the L858R mutation groups, and 0.84 , 0.77 and $0.85,0.69$ in the 19Del groups, respectively in the training and test cohorts. DCA showed that nomogram models have more benefits than ML models.

Conclusion: We constructed two predictive models for EGFR mutation status and subtypes. The nomogram models had more clinical usefulness than ML models.

\section{Introduction}

Lung cancer is one of the cause of cancer-related deaths, in particular, non-small cell lung cancer (NSCLC) accounts over $80 \%$ of all lung cancers [1]. Adenocarcinoma is the primary histological subtype of NSCLC [2]. Radiation therapy and/or chemotherapy are the treatment of choice for NSCLC; however, molecularly targeted drugs have been widely used in recent years. Epidermal growth factor receptor tyrosine kinase inhibitors (EGFR-TKIs) have been used in patients with lung adenocarcinoma with EGFR mutations $[3,4]$. EGFR-TKIs have demonstrated longer progression-free survival (PFS) than conventional chemotherapy $[5,6]$. Moreover, the regulation of EGFR mutations by EGFR-TKIs increases radiosensitivity [7]. However, dose escalation of radiation therapy does not improve the overall survival (OS) of patients with EGFR mutations [8]. Therefore, EGFR mutations identification is crucial for planning appropriate radiation treatment and other treatment regimens.

Among the EGFR mutation subtypes, exon 19 deletion mutation (19Del) and exon 21 point mutation (L858R) account for approximately $90 \%$ of all EGFR mutations $[3,9]$. These mutations are sensitive to EGFR-TKIs. However, 19Del and L858R exhibit varied characteristics, and 19Del show a better response to EGFR-TKIs than L858R[10]. Patients with 19Del also have a longer PFS than patients with L858R after treatment with EGFR-TKIs $[11,12]$. Therefore, identifying the mutation subtypes is critical for administering appropriate treatment and personalized therapy. 
EGFR mutations are typically detected using biopsy or surgical specimens [13]. However, these processes are time-consuming, expensive, and invasive for patients. Tumor heterogeneity also presents accurately detecting EGFR mutations from tumor-derived tissues [14]. Computed tomography (CT) is a useful tool for the non-invasive diagnosis and analysis of lung cancers $[15,16]$. Some studies have demonstrated using the use of radiomic features of lung tumors on CT images to predict EGFR mutation status and subtypes and/or OS by using machine learning (ML) models [9, 17-19]. Radiomics analyses the tumor phenotypes by automatically extracting numerous quantitative features (e.g., size, shape, texture, and histogram) from medical images [15, 20,21]. Its advantages of include its non-invasive approach and tumor visibility. Hong et al. reported the clinical effectiveness of nomogram by combining with radiomic features for EGFR mutation status [18]. Furthermore, Zhao et al. reported that radiomics-based nomograms could predict EGFR mutation subtype [22]. However, most of studies evaluated only ML models or nomogram models, and few studies compared ML models and nomogram models in the same data set for clinical usefulness. The superiority of the performance of $M L$ and nomogram models may depend on the prediction group.

The purpose of this study is to develop the predictive models for EGFR mutation status (EGFR mutation (EGFR+) vs. EGFR wild-type (EGFR-) groups) and subtypes (L858R vs. EGFR- groups and 19Del vs. EGFRgroups) by using ML models and nomogram, then validate the clinical effectiveness of these models.

\section{Materials And Methods}

\section{Patient data}

This study was approved by the Institutional Review Board of authors' affiliation (\#2020-148). A total of 172 patients with NSCLC who had undergone biopsy or surgical specimens between 2016 and 2020 from authors' affiliation were enrolled. The inclusion criteria were as follows: (a) pathologically confirmed as adenocarcinoma, (b) confirmed EGFR mutation status (mutation or wild-type), (c) confirmed EGFR mutation subtypes (L858R or 19Del or else), and (d) non-contrast enhanced chest CT images acquired before surgery, radiation therapy, chemotherapy, and/or targeted molecular therapy. The exclusion criteria were as follows: (a) patients who had tumors other than adenocarcinoma, (b) patients who had previously undergone surgery or targeted molecular therapy, and (c) simultaneous 19Del and L858R mutations. Patients who met the inclusion criteria were divided into two groups EGFR+ vs. EGFR-, L858R vs. EGFR- and 19Del vs. EGFR- in both the training and test cohorts. Fifteen patients with EGFR+ had subtypes other than 19Del and L858R. These subtypes were not included in subtype groups. The data were randomly divided into training and test cohorts in a ratio of 7:3. Clinical features included age, sex, location of lung tumor, smoking status, staging, c-reactive protein level, carcinoembryonic antigen level, cytokeratin 19 fragment level and chronic obstructive pulmonary disease status. The lung tumor location was divided into five lesions: right upper lobe, right middle lobe, right lower lobe, left upper lobe, and left lower lobe [2,17]. Fig. 1 shows the workflow of this study.

\section{CT imaging}


CT examinations were performed on multiple CT scanners, including Aquilion Precision (Canon Medical Systems, Otawara, Japan), Optima CT 660 (GE Healthcare, Waukesha, WI, USA), SOMATOM Sensation 64, SOMATOM Force, and SOMATOM Drive (Siemens Healthcare, Forchheim, Germany). The scanning parameters were as follows: tube voltage, $70-120 \mathrm{kV}$; tube current, $44-2585 \mathrm{~mA}$; slice thickness, 1.00 or $1.25 \mathrm{~mm}$; and field of view, $270-400 \mathrm{~mm}$. All the CT images were acquired from patients in the supine position, with both hands raised while inhaling. The $\mathrm{CT}$ images were converted to an isotropic volume of $1.00 \mathrm{~mm}$ using linear interpolation.

\section{Extraction of radiomic features and feature selection}

The lung tumors in the CT images were segmented semi-automatically using GrowCut in the open-source software 3D Slicer (version 4.10.2, Brigham and Women's Hospital) [22,23]. GrowCut segmentation for NSCLC showed a high correlation with the pathology [23]. Observers read CT images on the axial, coronal, and sagittal views using the mediastinum (width, $350 \mathrm{HU}$; level, $40 \mathrm{HU}$ ) and lung window (width, $1500 \mathrm{HU}$; level, $-500 \mathrm{HU}$ ) settings. Segmented tumors were used to extract the radiomic features using the opensource software Pyradiomics (version 3.7.1) in Python [24]. In total, 1046 radiomic features were extracted from the segmented tumors, including first-order, shape, gray-level co-occurrence matrix, graylevel run length matrix, gray-level size zone matrix, gray-level dependence matrix, Laplacian of Gaussian filters, gradient, and wavelet.

After feature extraction, all the features were standardized to eliminate the effects due to different CT scanners. To reduce the redundant features, selectKbest method in scikit-learn package which are based on analysis of variance and the least absolute shrinkage and selection operator (LASSO) were applied to training cohorts $[9,18]$. Five-fold cross-validation (CV) was applied to determine the tuning parameter that regularizes the magnitude of the penalization, and then features with non-zero coefficients were selected. Radiomics score (rad-score) was calculated through a linear combination of selected features multiplied by their coefficients $[18,22]$.

\section{Construction of machine learning and nomogram predictive models}

After rad-score was calculated, clinical features which showed significant difference were added. Then, two ML models (support vector machine (SVM) and logistic regression (LR)) were used to construct the prediction model for the three groups (rad-score alone, clinical features alone, rad-score + clinical features). In the SVM model, the radial basis function was applied and, in the both models, the grid search method with a five-fold CV was applied to optimize the hyperparameters.

Multivariable logistic analysis was performed to construct the nomogram. Clinical features and rad-score were included to select the independent features that predict EGFR mutation status or subtypes in the training cohort. Then, independent features were selected to construct the nomogram.

\section{Statistical analysis}


In the clinical data, quantitative data was assessed by using Student's t-test, and categorical data were assessed by using ${ }^{2}$ test. Rad-score was compared by using the Wilcoxon rank-sum test. The prediction performance of each ML model was evaluated in the area under the curve (AUC) of the receiver operating characteristic (ROC) curves in five-fold CV. Then, trained models were evaluated by the test cohorts. In the nomogram model, the concordance index (C-index) was calculated to quantify the discriminative performance of the nomogram [18][22]. Since C-index in the LR model is equal to the AUC [25], we evaluated the $\mathrm{C}$-index same value as the AUC. The Hosmer-Lemeshow $(\mathrm{HL})$ test was performed to evaluate the goodness-of-fit of logistic regression for the nomogram. Finally, the decision curve analysis (DCA) was performed to evaluate the clinical usefulness of the ML model, which showed the highest AUC in the each group and nomogram models in both cohorts [26]. When $p<0.05$, it is considered as a significant difference. All the procedures were performed by using in-house programs (Python ver. 3.7.1, $\mathrm{R}$ ver. 4.1.1).

\section{Results}

The clinical data of both cohorts are listed in Supplement Table 1, 2, and 3. There was a significant difference in sex and smoking in training cohorts of EGFR mutation status and subtypes. Furthermore, there was a significant difference in age in training cohorts in EGFR+ vs. EGFR-groups. In the training cohorts, seven, ten and five radiomic features were selected in EGFR+ vs. EGFR- groups, L858R vs. EGFRgroups, and 19Del vs. EGFR- groups, respectively through the feature selection. As shown in Supplement Tables 1, 2, and 3, the rad-score showed significant differences in both cohorts except test cohort in 19Del vs. EGFR- groups $(p=0.447)$. 
Table 1

The area under the curves and confidence intervals for each group in the two machine learning models.

\begin{tabular}{|c|c|c|c|c|c|c|}
\hline \multirow[t]{2}{*}{ Prediction group } & \multirow[t]{2}{*}{ Model } & \multirow[t]{2}{*}{ Signature } & \multicolumn{2}{|c|}{ Train cohort } & \multicolumn{2}{|c|}{ Test cohort } \\
\hline & & & AUC & {$[95 \% \mathrm{Cl}]$} & AUC & $\begin{array}{l}{[95 \%} \\
\mathrm{Cl}]\end{array}$ \\
\hline \multirow[t]{6}{*}{$\begin{array}{l}\text { EGFR mutation vs. EGFR } \\
\text { wild-type }\end{array}$} & \multirow[t]{3}{*}{ SVM } & Rad-score & $\begin{array}{l}0.706 \pm \\
0.088\end{array}$ & $\begin{array}{l}{[0.501} \\
0.913]\end{array}$ & 0.667 & $\begin{array}{l}{[0.518} \\
0.815]\end{array}$ \\
\hline & & Clinical & $\begin{array}{l}0.745 \pm \\
0.059\end{array}$ & $\begin{array}{l}{[0.548} \\
0.943]\end{array}$ & 0.670 & $\begin{array}{l}{[0.523} \\
0.818]\end{array}$ \\
\hline & & $\begin{array}{l}\text { Rad-score + } \\
\text { Clinical }\end{array}$ & $\begin{array}{l}0.775 \pm \\
0.072\end{array}$ & $\begin{array}{l}{[0.590} \\
0.960]\end{array}$ & 0.729 & $\begin{array}{l}{[0.590} \\
0.867]\end{array}$ \\
\hline & \multirow[t]{3}{*}{ LR } & Rad-score & $\begin{array}{l}0.705 \pm \\
0.088\end{array}$ & $\begin{array}{l}{[0.499} \\
0.912]\end{array}$ & 0.667 & $\begin{array}{l}{[0.518} \\
0.815]\end{array}$ \\
\hline & & Clinical & $\begin{array}{l}0.771 \pm \\
0.034\end{array}$ & $\begin{array}{l}{[0.581} \\
0.963]\end{array}$ & 0.664 & $\begin{array}{l}{[0.516} \\
0.813]\end{array}$ \\
\hline & & $\begin{array}{l}\text { Rad-score + } \\
\text { Clinical }\end{array}$ & $\begin{array}{l}0.818 \pm \\
0.031\end{array}$ & $\begin{array}{l}{[0.649} \\
0.990]\end{array}$ & 0.732 & $\begin{array}{l}{[0.594} \\
0.870]\end{array}$ \\
\hline \multirow[t]{6}{*}{ L858R vs. EGFR wild-type } & \multirow[t]{3}{*}{ SVM } & Rad-score & $\begin{array}{l}0.799 \pm \\
0.090\end{array}$ & $\begin{array}{l}{[0.561} \\
0.990]\end{array}$ & 0.764 & $\begin{array}{l}{[0.587} \\
0.942]\end{array}$ \\
\hline & & Clinical & $\begin{array}{l}0.761 \pm \\
0.177\end{array}$ & $\begin{array}{l}{[0.532} \\
0.946]\end{array}$ & 0.694 & $\begin{array}{l}{[0.501} \\
0.887]\end{array}$ \\
\hline & & $\begin{array}{l}\text { Rad-score + } \\
\text { Clinical }\end{array}$ & $\begin{array}{l}0.821 \pm \\
0.171\end{array}$ & $\begin{array}{l}{[0.665} \\
0.967]\end{array}$ & 0.804 & $\begin{array}{l}{[0.638} \\
0.971]\end{array}$ \\
\hline & \multirow[t]{3}{*}{ LR } & Rad-score & $\begin{array}{l}0.799 \pm \\
0.090\end{array}$ & $\begin{array}{l}{[0.561} \\
0.990]\end{array}$ & 0.764 & $\begin{array}{l}{[0.589} \\
0.942]\end{array}$ \\
\hline & & Clinical & $\begin{array}{l}0.760 \pm \\
0.210\end{array}$ & $\begin{array}{l}{[0.542} \\
0.931]\end{array}$ & 0.741 & $\begin{array}{l}{[0.557} \\
0.925]\end{array}$ \\
\hline & & $\begin{array}{l}\text { Rad-score + } \\
\text { Clinical }\end{array}$ & $\begin{array}{l}0.830 \pm \\
0.170\end{array}$ & $\begin{array}{l}{[0.668} \\
0.964]\end{array}$ & 0.826 & $\begin{array}{l}{[0.667} \\
0.985]\end{array}$ \\
\hline \multirow[t]{3}{*}{ 19Del vs. EGFR wild-type } & \multirow[t]{3}{*}{ SVM } & Rad-score & $\begin{array}{l}0.673 \pm \\
0.199\end{array}$ & $\begin{array}{l}{[0.394} \\
0.908]\end{array}$ & 0.587 & $\begin{array}{l}{[0.362} \\
0.811]\end{array}$ \\
\hline & & Clinical & $\begin{array}{l}0.706 \pm \\
0.164\end{array}$ & $\begin{array}{l}{[0.431} \\
0.945]\end{array}$ & 0.598 & $\begin{array}{l}{[0.373} \\
0.822]\end{array}$ \\
\hline & & $\begin{array}{l}\text { Rad-score + } \\
\text { Clinical }\end{array}$ & $\begin{array}{l}0.839 \pm \\
0.126\end{array}$ & $\begin{array}{l}{[0.640} \\
0.994]\end{array}$ & 0.773 & $\begin{array}{l}{[0.578} \\
0.970]\end{array}$ \\
\hline
\end{tabular}

Abbreviations: EGFR, epidermal growth factor receptor; SVM, support vector machine; LR, logistic regression; AUC, area under the curve; $\mathrm{Cl}$, confidence interval; Rad-score, radiomic score; L858R, exon 21 point mutation; 19Del, exon 19 deletion mutation. 


\begin{tabular}{|c|c|c|c|c|c|}
\hline \multirow[t]{3}{*}{ LR } & Rad-score & $\begin{array}{l}0.750 \pm \\
0.078\end{array}$ & $\begin{array}{l}{[0.466} \\
0.991]\end{array}$ & 0.587 & $\begin{array}{l}{[0.362} \\
0.812]\end{array}$ \\
\hline & Clinical & $\begin{array}{l}0.733 \pm \\
0.182\end{array}$ & $\begin{array}{l}{[0.473} \\
0.948]\end{array}$ & 0.713 & $\begin{array}{l}{[0.502} \\
0.924]\end{array}$ \\
\hline & $\begin{array}{l}\text { Rad-score + } \\
\text { Clinical }\end{array}$ & $\begin{array}{l}0.839 \pm \\
0.126\end{array}$ & $\begin{array}{l}{[0.640} \\
0.994]\end{array}$ & 0.773 & $\begin{array}{l}{[0.578} \\
0.970]\end{array}$ \\
\hline
\end{tabular}

Abbreviations: EGFR, epidermal growth factor receptor; SVM, support vector machine; LR, logistic regression; AUC, area under the curve; Cl, confidence interval; Rad-score, radiomic score; L858R, exon 21 point mutation; 19Del, exon 19 deletion mutation.

Table 2

The C-index and confidence intervals for each group in the nomogram model.

\begin{tabular}{|c|c|c|c|c|}
\hline \multirow[t]{2}{*}{ Prediction group } & \multicolumn{2}{|c|}{ Train cohort } & \multicolumn{2}{|c|}{ Test cohort } \\
\hline & C-index & {$[95 \% \mathrm{Cl}]$} & C-index & {$[95 \% \mathrm{Cl}]$} \\
\hline EGFR mutation vs. EGFR wild-type & 0.844 & {$[0.7760 .912]$} & 0.781 & {$\left[\begin{array}{ll}0.651 & 0.910\end{array}\right]$} \\
\hline L858R vs. EGFR wild-type & 0.833 & {$\left[\begin{array}{lll}0.723 & 0.942\end{array}\right]$} & 0.801 & {$\left[\begin{array}{lll}0.648 & 0.954\end{array}\right]$} \\
\hline 19Del vs. EGFR wild-type & 0.850 & {$\left[\begin{array}{lll}0.752 & 0.948\end{array}\right]$} & 0.689 & {$\left[\begin{array}{llll}0.454 & 0.924]\end{array}\right]$} \\
\hline
\end{tabular}

The AUCs and confidence intervals for each group in the two ML models are shown in Table 1. The models constructed by rad-score with clinical features showed the best AUC for both cohorts in all groups. In EGFR+ vs. EGFR- groups, LR showed the best AUC ( 0.818 for the training cohort and 0.732 for the test cohort). In L858R vs. EGFR- groups, LR showed the best AUC ( 0.830 for the training cohort and 0.826 for the test cohort). In 19Del vs. EGFR- groups, SVM and LR models showed the same AUC (0.839 for the training cohort and 0.773 for the test cohort).

Figure 2 shows the nomograms in the training cohort in each group. The $\mathrm{C}$-indices and confidence intervals for each group are shown in Table 2. The $\mathrm{C}$-indices of nomograms for the training and test cohort were 0.844 and 0.781 in EGFR+ vs. EGFR- groups, 0.833 and 0.801 in L858R vs. EGFR- groups, and 0.850 and 0.689 in 19Del vs. EGFR- groups, respectively. The $\mathrm{H}-\mathrm{L}$ test showed no significant difference in training and test cohorts in all models $(p=0.658, p=0.926$ in EGFR+ vs. EGFR-groups, $p=0.092, p=$ 0.326 in L858R vs. EGFR- groups, $p=0.346, p=0.190$ in 19Del vs. EGFR- groups, respectively), where these results mean that the logistic regression models have well goodness of fit [27]. Fig. 3 shows the decision curves of rad-score alone, clinical feature alone and nomogram (combined rad-score and clinical features) in each group. Those three models showed more net benefit than using the treat-all or the treatnone in both cohorts. Since the net benefit in DCA shows the clinical utility [28], we can consider all of those models have clinical usefulness. In addition, these curves mean that radiomic based nomogram has more net benefit than rad-score alone. Particularly, for risk threshold over $20 \%$, the nomogram model 
in each group has more benefit than that of the treat all and the treat none. Fig. 4 shows the decision curves of the best ML model and nomogram in each group. This showed that nomogram had more benefits than the best ML model in each group. For the risk threshold over $25 \%$, the nomogram model and best $M L$ model in each group have more benefits than that of the treat all and the treat none. Furthermore, for the risk threshold over $15 \%$, the nomogram model has more benefit than the ML model in each group in the training cohort. In the test cohort, though net benefits in two models were almost equal in L858R vs. EGFR- groups, those of nomogram models were slightly higher than ML models' in EGFR+ vs. EGFRgroups and 19Del vs. EGFR- groups.

\section{Discussion}

In this study, we constructed ML and nomogram models for predicting EGFR mutation status and subtypes. In the best ML model, for the EGFR+ vs. EGFR- groups, the AUC for the test cohort was 0.73 (SVM). Li et al. and Mei et al reported AUC of 0.79 and $0.66[9,19]$.The AUC of test cohorts of the L858R vs. EGFR- groups and the 19Del vs. EGFR- groups were 0.83 (LR) and 0.77 (SVM), respectively. Liu et al. reported that the AUC of L858R vs. EGFR- groups and 19Del vs. EGFR- groups were 0.92 and 0.77 , respectively, using positron emission tomography (PET) / CT images [13]. Though we cannot directly compare our AUC results due to different modalities, our models are comparable. The AUC of the 19Del vs. EGFR- groups in the test cohort was relatively low (0.77) than other groups because of the nonsignificant difference of rad-score. However, the AUC was the same value as the model of Li et al. though they did not use radiomic features as rad-score. Above these results, our ML models were reasonable results!

Hong et al. reported that the C-index of the nomogram model for EGFR+ vs. EGFR-groups by using contrast enhanced (CE) CT images was 0.83 in the test cohort [18]. On the other hand, the AUC of our model was 0.78; however, it is the results from plain CT images which have less information than CE CT images. It is, therefore, thought that our nomogram model was reasonable. Some studies reported $\mathrm{ML}$ models alone [9, 13, 19], and some studies reported nomogram models alone [22, 26]. However, few studies that compare models in the same data set have been reported. In this study, we compared $\mathrm{ML}$ models and nomogram models, then evaluated clinical effectiveness. Though the AUCs were almost equal ML and nomogram on most models, the ML model showed higher AUC in the 19Del vs. EGFRgroups in the test cohort and the nomogram model showed higher AUC in the EGFR+ vs. EGFR-groups in the test cohort. However, the results of DCA showed that the nomogram models have better clinical effectiveness than ML models. The model with higher net benefit has more clinical utility; therefore, clinicians can refer to these results to determine whether clinical decision machining based on our models will be useful or not [28]. Moreover, nomogram has the advantage as a simple visualization tool for calculating patient risk. Therefore, nomograms are considered a meaningful tool for clinical usage. Although smoking status contributes to the construction of our nomograms, Mitsudomi et al. reported that EGFR mutations are more common in non-smokers [6], indicating that there is no contradiction. Therefore, it may be possible to identify EGFR mutation and subtypes based on our nomograms. The nomogram demonstrated by Hong et al. consisted of sex; however, it did not contribute to our 
nomograms [18]. One of the reasons, may be the difference between the data set and the radiomics features that consist of the rad-score.

The gold standard for detecting EGFR mutation status detection is biopsy or surgical specimens. However, biopsy or surgical specimens could lead to incorrect diagnosis since the samples are often insufficient for analyzing the EGFR mutation status $[14,17]$. Therefore, combining radiomics with biopsy or surgical specimens results could allow more accurate identification of the EGFR mutation status and subtypes.

It is reported that patients who administered EGFR-TKIs have demonstrated longer PFS than conventional chemotherapy $[5,6]$. Moreover, it is reported that 19Del and L858R exhibit varied characteristics, and 19Del shows a better response to EGFR-TKIs than L858R [10]. Furthermore, the patients with 19Del have a longer PFS than patients with L858R after treatment with EGFR-TKIs $[11,12]$. Therefore, it is necessary to identify the EGFR mutation status and subtypes for appropriate selection of EGFR-TKI, and our prediction models are meaningful.

Our study has certain limitations. Only a few patients were included in this study; in particular, the sample size of cases with 19Del and L858R mutation subtypes was small. Therefore, a greater number of cases should be examined to improve the validity of our results. In addition, we did not validate our proposed nomograms with the external data set; it is necessary to compare with other models. Second, the characteristic of our used clinical data was limited. For example, we treated smoking status as "Yes" or "No" in this study, not like smoking habits. Furthermore, we did not use pathological data. For further study, more clinical information to improve the efficiency of our models will be added.

\section{Conclusions}

We constructed prediction models for EGFR mutation status and subtypes based on ML and nomogram by using radiomic features, then compared these models. The nomogram models showed more net benefit in the DCA. The nomogram models may have more clinical usefulness for predicting EGFR mutation status and subtypes than ML models.

\section{Declarations}

\section{Funding}

This study was supported by the Japan Society for the Promotion of Science (JSPS) KAKENHI Grant number 20K16789 (KF), 18 K07753 (TS), and the Takeda Science Foundation.

\section{Competing interests}

The authors have no relevant financial or non-financial interests to disclose.

\section{Author contributions}


Yusuke Kawazoe and Takehiro Shiinoki carried out the experiment. Yusuke Kawazoe wrote the manuscript with support from Takehiro Shiinoki, Koya Fujimoto, Yuki Yuasa. Tsunahiko Hirano and Kazuto Matsunaga supplied available data in terms of this study and discussed. Hidekazu Tanaka supervised this study. All authors discussed the results and contributed to the final manuscript.

\section{Conflicts of interest}

We have nothing conflicts of interest in this study.

\section{Ethical approval}

All procedures performed in studies involving human participants were in accordance with the ethical standards of the institutional and/or national research committee and with the 1964 Helsinki declaration and its later amendments or comparable ethical standards. This study was approved by the Institutional Review Board of Yamaguchi University, Japan. The ethics certificate number was \#2020-148.

\section{Consent to participants}

This study was retrospective study. We applied opt-out method to obtain consent on this study. The optout method was approved by the Institutional Review Board of Yamaguchi University.

\section{References}

1. Goldstraw P, Ball D, Jett JR, Le Chevalier T, Lim E, Nicholson AG et al (2011) Non-small-cell lung cancer. Lancet 378:1727-1740. https://doi.org/10.1016/S0140-6736(10)62101-0

2. Rizzo S, Petrella F, Buscarino V, De Maria F, Raimondi S, Barberis M et al (2016) CT radiogenomic characterization of EGFR, K-RAS, and ALK mutations in non-small cell lung cancer. Eur Radiol 26:3242. https://doi.org/10.1007/s00330-015-3814-0

3. Mitsudomi T, Yatabe Y (2007) Mutations of the epidermal growth factor receptor gene and related genes as determinants of epidermal growth factor receptor tyrosine kinase inhibitors sensitivity in lung cancer. Cancer Sci 98:1817-1824. https://doi.org/10.1111/j.1349-7006.2007.00607.x

4. Jänne PA, Engelman JA, Johnson BE (2005) Epidermal growth factor receptor mutations in nonsmall-cell lung cancer: implications for treatment and tumor biology. J Clin Oncol 23:3227-3234. https://doi.org/10.1200/JC0.2005.09.985

5. Maemondo M, Inoue A, Kobayashi K, Sugawara S, Oizumi S, Isobe H et al (2010) Gefitinib or chemotherapy for non-small-cell lung cancer with mutated EGFR. N Engl J Med 362:2380-2388. https://doi.org/10.1056/nejmoa0909530

6. Mitsudomi T, Morita S, Yatabe Y, Negoro S, Okamoto I, Tsurutani J et al (2010) Gefitinib versus cisplatin plus docetaxel in patients with non-small-cell lung cancer harbouring mutations of the epidermal growth factor receptor (WJTOG3405): an open label, randomised phase 3 trial. Lancet Oncol 11:121-128. https://doi.org/10.1016/S1470-2045(09)70364-X 
7. Kriegs M, Gurtner K, Can Y, Brammer I, Rieckmann T, Oertel R et al (2015) Radiosensitization of NSCLC cells by EGFR inhibition is the result of an enhanced p53-dependent G1 arrest. Radiother Oncol 115:120-127. https://doi.org/10.1016/j.radonc.2015.02.018

8. Bradley JD, Paulus R, Komaki R, Masters G, Blumenschein G, Schild S et al (2015) Standard-dose versus high-dose conformal radiotherapy with concurrent and consolidation carboplatin plus paclitaxel with or without cetuximab for patients with stage IIIA or IIIB non-small-cell lung cancer (RTOG 0617): A randomised, two-by-two factorial p. Lancet Oncol 16:187-199. https://doi.org/10.1016/S1470-2045(14)71207-0

9. Li S, Luo T, Ding C, Huang Q, Guan Z, Zhang H (2020) Detailed identification of epidermal growth factor receptor mutations in lung adenocarcinoma: combining radiomics with machine learning. Med Phys 47:3458-3466. https://doi.org/10.1002/mp.14238

10. Soria JC, Mok TS, Cappuzzo F, Jänne PA (2012) EGFR-mutated oncogene-addicted non-small cell lung cancer: current trends and future prospects. Cancer Treat Rev 38:416-430. https://doi.org/10.1016/j.ctrv.2011.10.003

11. Lee CK, Davies L, Wu YL, Mitsudomi T, Inoue A, Rosell R et al (2017) Gefitinib or erlotinib vs chemotherapy for EGFR mutation-positive lung cancer: individual patient data meta-analysis of overall survival. J Natl Cancer Inst 109:1-9. https://doi.org/10.1093/jnci/djw279

12. Sutiman N, Tan SW, Tan EH, Lim WT, Kanesvaran R, Ng QS et al (2017) EGFR mutation subtypes influence survival outcomes following first-line gefitinib therapy in advanced Asian NSCLC patients. J Thorac Oncol 12:529-538. https://doi.org/10.1016/j.jtho.2016.11.2225

13. Liu Q, Sun D, Li N, Kim J, Feng D, Huang G et al (2020) Predicting EGFR mutation subtypes in lung adenocarcinoma using 18F-FDG PET/CT radiomic features. Transl Lung Cancer Res 9:549-562. https://doi.org/10.21037/tlcr.2020.04.17

14. Zhang L, Zhang Y, Chang L, Yang Y, Fang W, Guan Y et al (2019) Intratumor heterogeneity comparison among different subtypes of non-small-cell lung cancer through multi-region tissue and matched ctDNA sequencing. Mol Cancer 18:1-6. https://doi.org/10.1186/s12943-019-0939-9

15. Gillies RJ, Kinahan PE, Hricak H (2016) Radiomics: images are more than pictures, they are data. Radiology 278:563-577. https://doi.org/10.1148/radiol.2015151169

16. Park H, Sholl LM, Hatabu H, Awad MM, Nishino M (2019) Imaging of precision therapy for lung cancer: current state of the art. Radiology 293:15-29. https://doi.org/10.1148/radiol.2019190173

17. Li S, Ding C, Zhang H, Song J, Wu L (2019) Radiomics for the prediction of EGFR mutation subtypes in non-small cell lung cancer. Med Phys 46:4545-4552. https://doi.org/10.1002/mp.13747

18. Hong D, Xu K, Zhang L, Wan X, Guo Y (2020) Radiomics signature as a predictive factor for EGFR mutations in advanced lung adenocarcinoma. Front Oncol 10:1-8. https://doi.org/10.3389/fonc. 2020.00028

19. Mei D, Luo Y, Wang Y, Gong J (2018) CT texture analysis of lung adenocarcinoma: can Radiomic features be surrogate biomarkers for EGFR mutation statuses. Cancer Imaging 18:1-9. https://doi.org/10.1186/s40644-018-0184-2

Page 11/14 
20. Lambin P, Rios-Velazquez E, Leijenaar R, Carvalho S, Van Stiphout RGPM, Granton P et al (2012) Radiomics: extracting more information from medical images using advanced feature analysis. Eur J Cancer 48:441-446. https://doi.org/10.1016/j.ejca.2011.11.036

21. Aerts HJWL, Velazquez ER, Leijenaar RTH, Parmar C, Grossmann P, Cavalho S et al (2014) Decoding tumour phenotype by noninvasive imaging using a quantitative radiomics approach. Nat Commun 5:1-9. https://doi.org/10.1038/ncomms5006

22. Zhao W, Wu Y, Xu Y, Sun Y, Gao P, Tan M et al (2020) The potential of radiomics nomogram in noninvasively prediction of epidermal growth factor receptor mutation status and subtypes in lung adenocarcinoma. Front Oncol 9:1485. https://doi.org/10.3389/fonc.2019.01485

23. Velazquez ER, Parmar C, Jermoumi M, Mak RH, Van Baardwijk A, Fennessy FM et al (2013) Volumetric CT-based segmentation of NSCLC using 3D-slicer. Sci Rep 3:1-7. https://doi.org/10.1038/srep03529

24. Van Griethuysen JJM, Fedorov A, Parmar C, Hosny A, Aucoin N, Narayan V et al (2017) Computational radiomics system to decode the radiographic phenotype. Cancer Res 77:e104-e107. https://doi.org/10.1158/0008-5472.CAN-17-0339

25. Pencina MJ, D’Agostino RB (2015) Evaluating discrimination of risk prediction models: The $C$ statistic. JAMA - J Am Med Assoc 314:1063-1064. https://doi.org/10.1001/jama.2015.11082

26. Huang Y, Liu Z, He L, Chen X, Pan D, Ma Z et al (2016) Radiomics signature: A potential biomarker for the prediction of disease-free survival in early-stage (I or II) non-small cell lung cancer. Radiology 281:947-957. https://doi.org/10.1148/radiol.2016152234

27. Kuang Y, Li R, Jia P, Ye W, Zhou R, Zhu R et al (2021) MRI-Based Radiomics: Nomograms predicting the short-term response after transcatheter arterial chemoembolization (TACE) in hepatocellular carcinoma patients with diameter less than $5 \mathrm{~cm}$. Abdom Radiol 46:3772-3789. https://doi.org/10.1007/s00261-021-02992-2

28. Zhang B, Liu Q, Zhang X, Liu S, Chen W, You J et al (2020) Clinical Utility of a Nomogram for Predicting 30-Days Poor Outcome in Hospitalized Patients With COVID-19: Multicenter External Validation and Decision Curve Analysis. Front Med 7:1-12. https://doi.org/10.3389/fmed.2020.590460

\section{Figures}




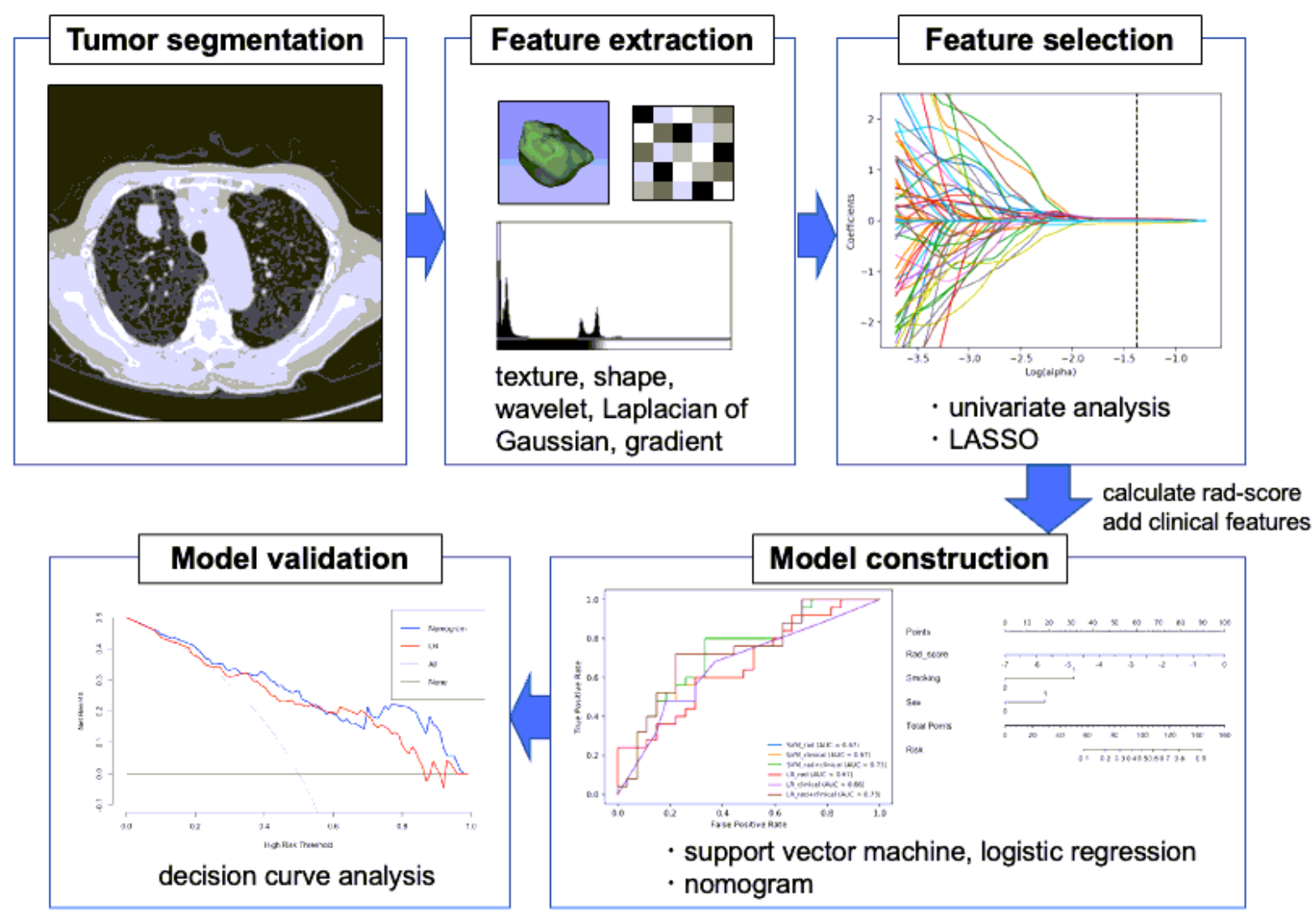

Figure 1

Study design and workflow

(a)

$$
\begin{aligned}
& \text { Points } \\
& \text { Rad_score } \\
& \text { Smoking } \\
& \text { Age } \\
& \text { Total Points }
\end{aligned}
$$

Risk

(c)

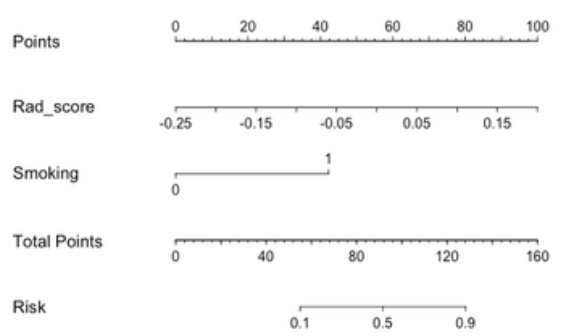

(b)

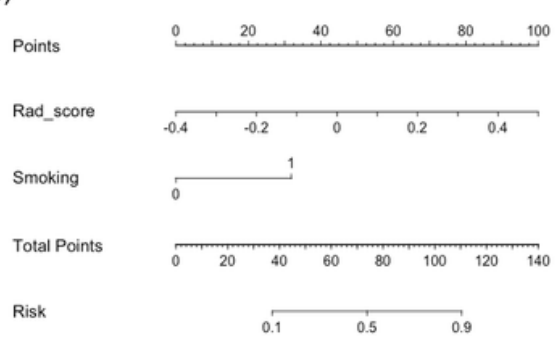




\section{Figure 2}

The nomograms in training cohort. (a) epidermal growth factor receptor (EGFR) mutation vs. wild-type groups. (b) exon 21-point mutations vs. wild-type groups. (c) exon19 deletions vs. wild-type groups.

\section{Figure 3}

The decision curve analysis (DCA) for each model

The $y$-axis means the net benefit. The net benefit $=$ true positive rate $-($ false positive rate $\times$ weighting factor), where the weighting factor $=$ threshold $-(1$ - threshold $)$. The nomogram model had the highest net benefit compared with radiomics alone or clinical feature alone and simple strategies such as gray line, which assumes that all tumors were epidermal growth factor receptor (EGFR) mutation (the treat all); horizontal black line which assumes that all tumors were EGFR wild-type (the treat none). (a) The DCA in the EGFR mutation vs. wild-type groups in the training (left) and test (right) cohorts. (b) The DCA in the exon 21-point mutations vs. wild-type groups in the training (left) and test (right) cohorts. (c) The DCA in the exon 19 deletions vs. wild-type groups in the training (left) and test (right) cohorts. For the risk threshold over $20 \%$, the nomogram model in each group has more benefits than the treat all and the treat none.

\section{Figure 4}

The decision curve analysis (DCA) for the best machine learning and nomogram model

(a) The DCA in the EGFR mutation vs. wild-type groups in the training (upper) and test (lower) cohorts. (b) The DCA in the exon 21-point mutations vs. wild-type groups in the training (upper) and test (lower) cohorts. (c) The DCA in the exon 19 deletions vs. wild-type groups in the training (upper) and test (lower) cohorts. For the training cohort, the nomogram model in each group has more benefit than that of the machine learning model. For the test cohort, the benefits of the two models are almost the same in the EGFR mutation vs. wild-type groups and the exon 21-point mutations vs. wild-type groups. However, in the exon 19 deletions vs. wild-type groups, the nomogram has more benefit than the machine learning model for the risk threshold of over $40 \%$. 